\title{
The role of NTHi colonization and infection in the pathogenesis of neutrophilic asthma
}

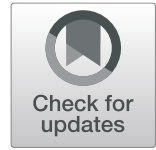

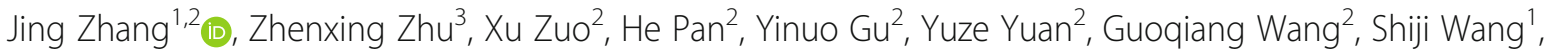
Ruipeng Zheng ${ }^{2,4}$, Zhongmin Liu', Fang Wang ${ }^{2}$ and Jingtong Zheng ${ }^{2,5^{*}}$ (D)

\begin{abstract}
Asthma is a complex heterogeneous disease. The neutrophilic subtypes of asthma are described as persistent, more severe and corticosteroid-resistant, with higher hospitalization and mortality rates, which seriously affect the lives of asthmatic patients. With the development of high-throughput sequencing technology, an increasing amount of evidence has shown that lower airway microbiome dysbiosis contributes to the exacerbation of asthma, especially neutrophilic asthma. Nontypeable Haemophilus influenzae is normally found in the upper respiratory tract of healthy adults and is one of the most common strains in the lower respiratory tract of neutrophilic asthma patients, in whom its presence is related to the occurrence of corticosteroid resistance. To understand the pathogenic mechanism by which nontypeable Haemophilus influenzae colonization leads to the progression of neutrophilic asthma, we reviewed the previous literature on nontypeable Haemophilus influenzae colonization and subsequent aggravation of neutrophilic asthma and corticosteroid resistance. We discussed nontypeable Haemophilus influenzae as a potential therapeutic target to prevent the progression of neutrophilic asthma.
\end{abstract}

Keywords: Neutrophilic asthma, Nontypeable Haemophilus influenzae (NTHi), Oxidative stress, Th17/Treg imbalance, Corticosteroid-resistant

\section{Background}

Asthma is a complex airway inflammatory disease. It has been reported that there are more than 300 million asthma patients worldwide. The incidence of asthma varies greatly among different countries and regions. In recent decades, the mortality rate of asthma has declined significantly worldwide (Fig. 1a), mainly due to the widespread use of inhaled corticosteroids (ICS). However, the number of new cases of asthma is still increasing (Fig. 1b). According to a prediction from the Global Initiative for Asthma (GINA), 400 million people worldwide will suffer from asthma by 2025. Asthma is considered to be

\footnotetext{
* Correspondence: wszjt1@163.com

${ }^{2}$ Department of Pathogen Biology, College of Basic Medical Sciences, Jilin University, Changchun 130021, China

${ }^{5}$ Key Laboratory of Zoonosis, Ministry of Education, College of Veterinary Medicine, Jilin University, Changchun 130062, China

Full list of author information is available at the end of the article
}

a major cause of disability, substantial medical expenditures and preventable death.

In the past, asthma was too often viewed as a monolithic entity and was known as a type 1 hypersensitivity disease with eosinophilic bronchitis. The Th2 response plays a significant role in asthma, leading to interleukin4 (IL-4), IL-5, and IL-13 production, IgE-mediated responses, mucus secretion and airway hyperreactivity (AHR) [1]. These are classical explanations of asthma. The view of asthma as a single entity model is now obsolete because people have a better understanding of the heterogeneity of asthma. The latest definition of asthma is based on a history of respiratory symptoms, such as wheezing, shortness of breath, chest tightness and cough, which vary with time and have varying intensity, and variable expiratory airflow restrictions. This clinical definition focuses on variable respiratory symptoms and variable airflow limitations, which are two key features 

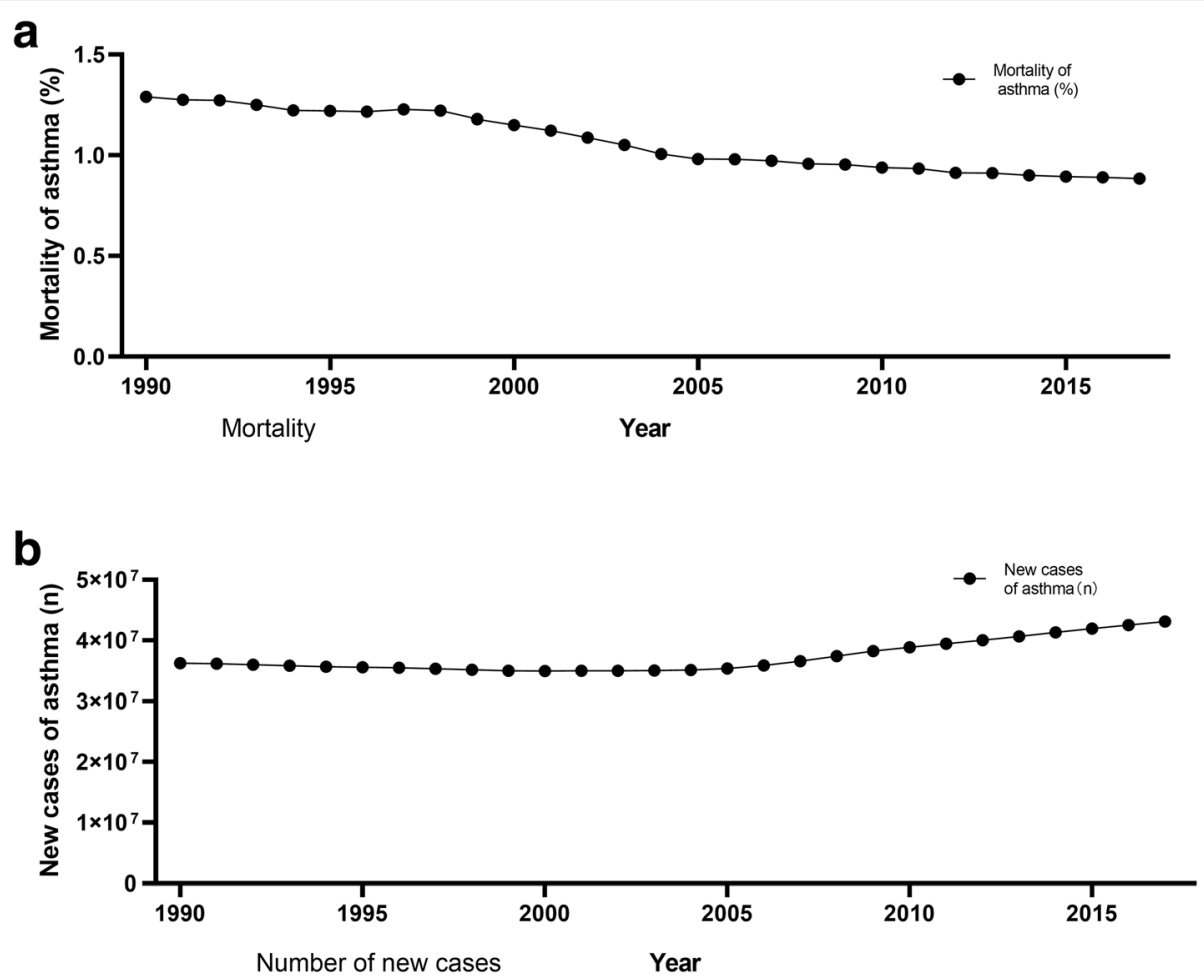

Fig. 1 Global mortality of asthma (a) and the number of new cases of asthma (b) between 1990 and 2017 among all ages. Created with data from the Global Burden of Disease Study 2017 (GBD 2017) Results

needed for an asthma diagnosis, rather than the pathological and physiological characteristics of asthma that were previously used [2].

Asthma can be divided into subtypes, which include eosinophilic asthma, neutrophilic asthma, mixed granulocytic asthma and paucigranulocytic asthma, according to the sputum cell count and classification [3]. While eosinophilic inflammation has been considered to be the hallmark of airway inflammation in asthma [2], it is present in only $50 \%$ of asthmatic patients [4]. Compared with eosinophilic asthma, neutrophilic asthma is described as persistent, more severe and corticosteroidresistant. Although neutrophilic asthma accounts for only $5-20 \%$ of all asthma cases, it consumes more than $50-80 \%$ of the medical resources related to asthma and has higher hospitalization and mortality rates, which seriously affect the lives of asthmatic patients and have also become a substantial burden on society and the public health system $[5,6]$. Therefore, neutrophilic asthma is currently the most perplexing and challenging presentation of "asthma".

Many theories have been proposed to explain the rising incidence of asthma. The most striking is Strachan's hygiene hypothesis, which holds that the increasingly clean and sterile environment in modern life promotes the development of many diseases, including asthma, suggesting that some bacteria may play a protective role in the occurrence of asthma [7]; however, animal model studies support the role of the microflora in the development of asthma and atopic diseases [8]. In recent years, with the development of microbial identification technologies (culture-independent technology), the identification of microbial species, especially bacteria, has become increasingly sensitive. The overall composition of microbial communities has been analyzed in detail [9]. Studies have confirmed that bacterial diversity and microbial community composition are related to the degree of AHR. The airway microbial diversity of patients with neutrophilic asthma decreases significantly. The increase in members of Haemophilus influenzae is associated with asthma and is related to the severity of this disease $[10,11]$.

Nontypeable Haemophilus influenzae (NTHi) is a noncapsular strain of Haemophilus influenzae and one of the main pathogenic bacteria of the respiratory tract. With the wide application of the Haemophilus influenzae type b conjugate vaccine [12], the incidence of NTHi is increasing annually [13]. Current studies have shown that NTHi is one of the most common bacteria isolated from asthmatic patients and may play an important role in the progression and worsening of asthma [14], especially neutrophilic asthma [15]. In this review, we focus on the structure and pathogenicity of NTHi and describe recent insights into the role of NTHi in neutrophilic 
asthma exacerbation. We will discuss the potential of treating NTHi infections and improving airway flora imbalances as a strategy for treating neutrophilic asthma and preventing its onset.

\section{NTHi structure and pathogenicity}

Haemophilus influenzae strains are divided into those with a capsule (e.g., the type b form) and those without a capsule (such as NTHi). The encapsulated strains primarily play roles in systemic infections in conditions such as meningitis, while NTHi strains rarely cause disease outside the respiratory tract and can be considered the main pathogen of the respiratory mucosa.

There is abundant evidence that NTHi is an intracellular pathogen. NTHi has a variety of structures, such as lipooligosaccharides (LOSs), outer membrane proteins, adhesions, pili, Hia and hap proteins, which can inhibit ciliary function and bind to mucus to help NTHi adhere to the respiratory mucosa [16-18]. This is the first step of NTHi colonization. At the same time, NTHi also has an excellent ability to escape mucosal immune mechanisms, including proteases, microflora and phase changes and antigenic drift [19-22]. After evading host mucosal immunity, NTHi can use its invasive ability to enter local respiratory tissues and survive in cells.

The mechanism through which NTHi enters host cells is very complex and includes actin, tubulin, the formation of a lamellar foot and microvilli, and the ingestion of bacteria into vesicles [23]. The most effective entry mechanism seems to be through the interaction of phosphatidylcholine residues on NTHi oligosaccharides with human platelet activating factor receptor (PAFR), as blocking PAFR with specific inhibitors can reduce the invasion of NTHi by more than $90 \%$ [24]. Other possible host pathways utilized by pathogens include macropinocytosis, phagocytosis, receptor-mediated endocytosis, lipid raft-mediated endocytosis, autophagy, secretion, transcytosis, and paracytosis [25]. The target cells of NTHi are mainly macrophages and airway epithelial cells [25], in which NTHi can escape the killing effects of antibiotics and bactericidal antibodies [23]. In addition, NTHi can evade the killing effects of the complement system in some unique ways, such as altering LOSs, sialic acid and other outer membrane proteins to suppress complement system activation, enhancing the effect of complement activation inhibitors such as $\mathrm{C} 4$ binding protein and factor $\mathrm{H}$ [26], inhibiting the function of lymphocytes [27], inhibiting the effects of IgA (important antibodies for killing NTHi via mucosal immunity) and downregulating the expression of IgA. Through these structures and functions, NTHi creates an opportunity to settle in the human respiratory tract for a long time without being cleared by the host immune system.

NTHi is a bacterium that is present in the nasopharynx of most healthy adults, and in this situation, it appears to be a commensal organism [28]. NTHi may also spread to the lower respiratory tract and has been suggested to be associated with many important diseases, such as otitis media, lower respiratory tract infections, the exacerbation of chronic obstructive pulmonary disease (COPD), bronchiectasis and cystic fibrosis [12, 26, 28]. Accumulating evidence suggests that the development of asthma is related to NTHi colonization. Specifically, NTHi infection in early life is closely related to the occurrence of childhood asthma. Recent animal experiments have confirmed this hypothesis. Infection with NTHi on the third day after birth in mice significantly increases the possibility of developing or aggravating asthma in infancy [4]. Hilty and colleagues have come to a similar conclusion for adults and found that Haemophilus is more likely to be present in the lower respiratory tract of adults with asthma than in patients with normal lung function [10]. In fact, NTHi is one of the most common bacteria isolated from the airway of patients with neutrophilic asthma [27]. However, it is not clear what role NTHi plays in the occurrence and deterioration of neutrophilic asthma. This review will summarize the previously published mechanisms through which NTHi may lead to the occurrence and aggravation of neutrophilic asthma and will speculate on the relationship between NTHi and neutrophilic asthma.

\section{Potential mechanisms by which NTHi leads to asthma Hypersecretion of mucus}

Mucus secretion and clearance in the normal airway mucosa are in a dynamic balance, which means that mucus not only protects the airway but also clears dust and pathogens in the inhalation airway without causing airway obstruction (Fig. 2a). The hypersecretion of mucus is one of the manifestations of acute asthma attacks and is one of the characteristics of airway remodeling in neutrophilic asthma. McCann et al. [4] observed that previously NTHi-colonized, allergen-sensitized mice secreted increased amounts of mucus into the airways [14]. Researchers have studied the mechanism by which NTHi infection induces excessive mucus secretion in airway epithelial cells. NTHi can strongly promote transcription of the mucin MUC5AC (MUC5AC is one of the main components of mucus) by upregulating the p38 mitogen-activated protein kinase (MAPK) pathway [29]. In addition, the secretion of MUC2 in asthma is also significantly increased, and because MUC2 is highly insoluble, even a small amount of MUC2 may lead to excessive airway mucus viscosity and airway obstruction 


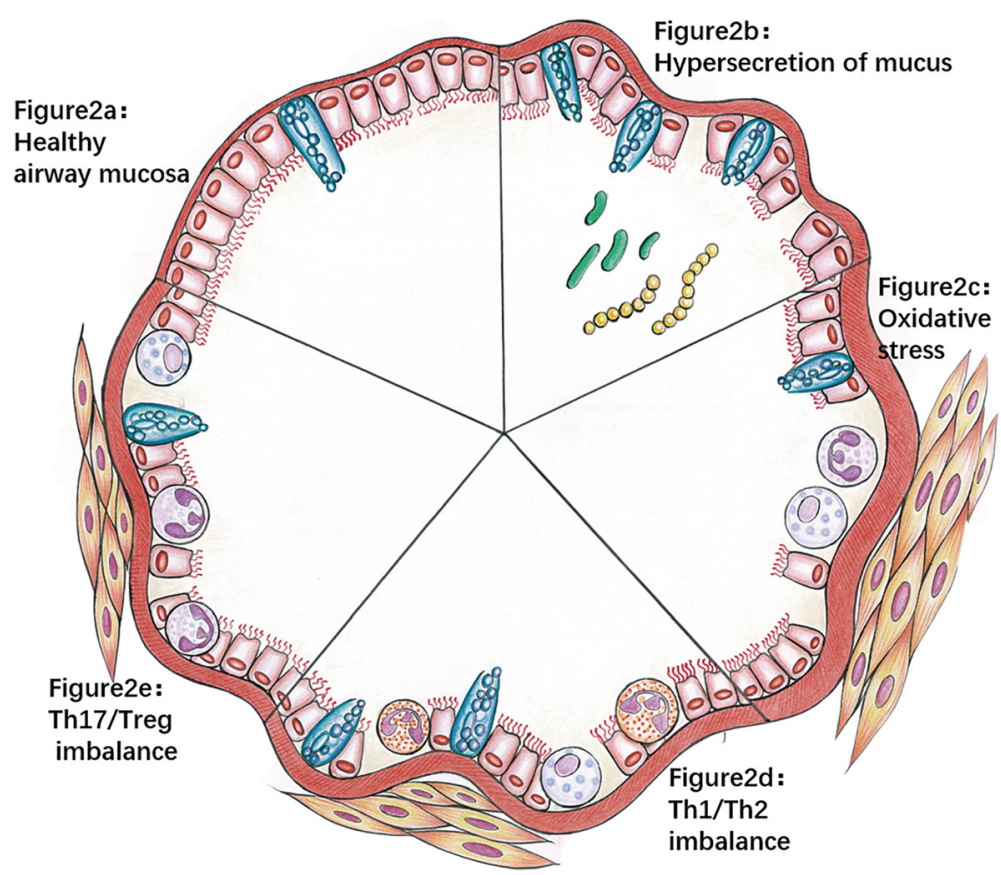

Fig. 2 Potential mechanisms by which NTHi leads to asthma. Healthy airways (a) have no inflammatory cell infiltration, and goblet cells secrete mucus to lubricate the airway. After NTHI infection: When large amounts of mucus are secreted (b), the airway is narrow and obstructed, and other bacteria may cause repeated infections, resulting in airway damage. When oxidative stress occurs, (c) neutrophils and mast cells accumulate in the airway, and mucus secretion increases, which is accompanied by airway remodeling and severe airway injury. When Th1/Th2 imbalance (d) occurs, the number of eosinophils and mast cells increases and mucus secretion increases, which is accompanied by airway remodeling and airway injury. When Th17/Treg imbalance occurs (e), a large number of neutrophils accumulate in the airway, and mucus secretion increases, which is accompanied by airway remodeling and airway injury

in asthma [30]. The mechanism of increased MUC2 secretion caused by NTHi infection is related to the transforming growth factor-beta-Smad signaling pathway in cooperation with nuclear factor-kappa B (NF-kB) [31]. The oversecretion of airway mucus can lead to airway stenosis, airflow obstruction and repeated respiratory tract infections (Fig. 2b). This may be one of the main mechanisms by which NTHi causes neutrophilic asthma to worsen.

\section{Oxidative stress}

Oxidative stress refers to the damage that occurs when reactive oxygen species (ROS) exceed the host's antioxidant defenses. Oxidative stress is one of the fatal causes of airway damage in chronic inflammatory diseases, such as COPD, bronchiectasis and neutrophilic asthma. A large amount of evidence has shown that oxidative stress is highly involved in the pathogenesis of human asthma and plays a key role in AHR, neutrophilic inflammation, airway remodeling, and even corticosteroid resistance [32]. Nuclear factor erythroid 2-related factor 2 (Nrf2) is involved in a classical antioxidant pathway. When Nrf2 gene dysfunction occurs, animal models are prone to asthma induction or asthma aggravation, and a large number of neutrophils aggregate, resulting in airway inflammation, AHR and airway remodeling [33, 34]. At the same time, the use of an Nrf2 activator or an antioxidant stress treatment can improve symptoms, which confirms the importance of oxidative stress in neutrophilic asthma [35].

When a patient is infected with NTHi, innate immunity and adaptive immunity are activated in many ways, and inflammatory cells (including neutrophils, epithelial cells, macrophages, etc.) are activated [26]. NTHi, as an intracellular pathogen, can significantly increase the production of ROS in human fibroblasts, epithelial cells, macrophages and neutrophils after airway infection. ROS production was the highest in neutrophils and macrophages, and this production continued with prolonged NTHi infection. Moreover, a study also confirmed that ROS production was related to the formation of neutrophil extracellular traps (NETs), and the addition of DNase significantly inhibited the production of extracellular traps [36].

To kill NTHi, inflammatory cells develop respiratory bursts. During respiratory bursts, inflammatory cells release high concentrations of $\mathrm{O}_{2^{-}}, \cdot \mathrm{OH}, \mathrm{HOCl} / \mathrm{HOBr}$ and $\mathrm{H}_{2} \mathrm{O}_{2}$, which can penetrate tissues and lead to increases in free radicals in surrounding tissues [32]. As a typical respiratory pathogen, NTHi has evolved a variety of 
defense mechanisms, such as catalase, peroxidase/glutamine enzyme and ferritin-like protein [37, 38]. This ability to resist oxidative stress is important for the colonization and pathogenicity of NTHi in the airway. The activation of excessive oxidative stress, such as through a signaling cascade, fails to kill NTHi but results in the imbalance of oxidants and antioxidants and may lead to cell damage through the oxidation of proteins, DNA and lipids, which has many adverse effects on the airway mucosa, including airway smooth muscle contraction, the induction of airway hyperresponsiveness, excessive mucus secretion, epithelial exfoliation, vascular exudation and airway remodeling [39, 40] (Fig. 2c). Furthermore, NTHi has a higher affinity for the damaged mucosa [41], leading to long-term colonization and chronic airway inflammation. There is a cycle of NTHi infection and oxidative stress: ROS induce the production of cytokines and chemokines by inducing oxidative stress-sensitive NF- $\mathrm{KB}$ transcription in bronchial epithelial cells, leading to further inflammation and more oxidative stress [42, 43]; this process further damages the airway mucosa and promotes the sustained colonization of NTHi. At the same time, sustained colonization promotes the progression of airway inflammation and the increase in oxidative stress. This process eventually leads to chronic airway inflammation and long-term, chronic colonization of NTHi.

Therefore, the level of oxidative stress in NTHiinfected asthmatic patients is high, which increases airway damage. Similarly, asthmatic patients with impaired airway barrier function are more vulnerable to oxidative stress caused by NTHi infection, which leads to the development of asthma.

\section{Th1/Th2 imbalance}

Numerous previous studies have confirmed that asthma is a chronic inflammatory airway disease mainly caused by the Th2 response. The excessive Th2 response leads to significantly increased levels of cytokines such as IL-14, IL-5, and IL-13, resulting in the aggregation of eosinophils in the airway, increased levels of IgE in the serum, and hyperresponsiveness of the airway [44] (Fig. 2d). It has been reported that the Th2 response was the main reaction in NTHi-colonized mice, and the Th1 response was inhibited, suggesting that chronic NTHi colonization may lead to an enhanced Th2 response. The Th1/Th2 imbalance hypothesis has been used to explain the pathogenesis of asthma in recent decades. In animal models of asthma, Th1/Th2 imbalances can be found, and some studies have tried to improve this imbalance to treat asthma [4, 45]. However, some clinical trials have yielded disappointing results, especially in the treatment of severe asthma, refractory asthma and glucocorticoid-resistant asthma [1, 46]. Over the past decade, the understanding of the pathogenesis of asthma has changed dramatically, from a single disease that is mediated by IgE and depends on Th2 cells to a more complex, heterogeneous disease. The Th1/Th2 imbalance hypothesis does not fully explain the pathogenesis of asthma, especially neutrophilic asthma [47, 48]. Th1/Th2 imbalances exist in neutrophilic asthma, but there must be other pathways beyond the Th2 pathway that play an important role in the pathogenesis of asthma, especially neutrophilic asthma. For example, strong Th17 responses can promote an increase in neutrophils in the airway and acute AHR, leading to acute asthma attacks [49]. In particular, the airway remodeling induced by Th17 cells is independent of the Th2 reaction and plays a crucial role in the pathogenesis of corticosteroid-resistant asthma and neutrophilic asthma [50]. It has been reported that NTHi-infected mice exhibit enhanced Th2 responses and suppressed Th1 responses, suggesting that NTHi infection may cause Th1/Th2 imbalances, but this is not the main mechanism for NTHirelated neutrophilic asthma [27].

\section{Th17/Treg imbalance}

Asthma is a chronic inflammatory disease of the airway. The $\mathrm{T}$ cell-mediated adaptive immune response plays a significant role in the occurrence and aggravation of neutrophilic asthma. Th17 and Treg cells are two important subsets of CD4+ T cells: Th17 cells can produce proinflammatory cytokines, of which IL-17 is a potent chemoattractant of neutrophils, which can mediate host defense against pathogens; Tregs play a negative regulatory role in immunity [51]. Research confirms that NTHi infection promotes the Th17 response and IL-17 secretion and inhibits the Treg response [4, 27]. As an inflammatory factor secreted by Th17 cells, IL-17A (one of the main members of the IL-17 family) plays a vital role in the occurrence of neutrophilic asthma, and the expression level of IL-17A is positively correlated with the severity of asthma [52, 53]. Th17/IL-17 can promote the activation of $\mathrm{T}$ cells, upregulate proinflammatory cytokines and chemokines, drive neutrophils to aggregate in the lungs, kill pathogenic bacteria, produce excessive airway neutrophilic inflammation, and damage the airway mucosa (Fig. 2e). This neutrophil inflammatory response can be reversed by enhancing Treg cell activity, suggesting that the imbalance of Th17/Treg cells may be a critical mechanism for the development of neutrophilic asthma.

In addition to causing airway neutrophilic inflammation, the Th17 response and elevated IL-17 can also promote airway remodeling, which is one of the most important features of asthma (Fig. 2e). Studies have shown that IL-17 can upregulate fibroblast growth factor and angiogenesis factor and can promote collagen synthesis [54]. At the same time, IL-17 is also believed to strongly promote the synthesis and secretion of the 
mucin MUC5AC (a major constituent of mucus) from goblet cells [55]. Moreover, IL-17 can also increase the proliferation and migration of human airway smooth muscle (ASM) cells through an ERK1/2-MAP kinasedependent pathway [56], and Th17 cells can also inhibit ASM cell apoptosis [57]. Interestingly, bronchial fibroblasts obtained from asthmatic patients can specifically promote Th17 cell differentiation in vitro [58], which may indicate that the local airway environment of asthmatic patients is conducive to Th17 cell differentiation.

Additional studies have shown that Th17/Treg imbalances may also explain NTHi-related corticosteroid resistance in neutrophilic asthma. In vitro, dexamethasone can inhibit eosinophils, but not neutrophils, and can even promote the development of Th17 cells [50]. More detailed information on Th17/Treg imbalances and NTHi-related corticosteroid resistance will be discussed later in this article.

In conclusion, under physiological conditions, the proinflammatory response of Th17 cells and the antiinflammatory response of Treg cells are in a state of balance, so that the immune response can eliminate pathogens without causing damage to the body. NTHi infection leads to the dominance of Th17 and its cytokines, can induce airway neutrophilic inflammation and can promote airway injury and airway remodeling, which is closely related to the development of neutrophilic asthma and may be a potential pathogenic mechanism of corticosteroid-resistant asthma.

\section{Other potential mechanisms}

The above mechanisms are possible mechanisms by which NTHi causes or promotes neutrophilic asthma. However, interestingly, in an ovalbumin (OVA)-sensitized model of asthma in mice, NTHi infection does not persist for more than 10 days but can promote asthma progression after 16 days [27]. Thus, the primary effect of this infection may be to induce persistent immune changes that persist even after the infection has been cleared, which behave synergistically with exposure to allergens to drive neutrophilic allergic airway diseases (AADs). Another mouse experiment also found that early exposure to NTHi significantly affected the incidence of anaphylactic airway diseases and the severity of AHR later in life [4]. A similar situation has been suggested in humans; children colonized with NTHi early in life were more likely to develop symptoms of asthma when they were older than children without detectable NTHi in the nasopharynx [59]. It has been speculated that NTHi colonization/infection may have a sustained impact on the immune system, contribute to the establishment of a proneutrophilic environment, and promote the occurrence and development of neutrophilic asthma in response to allergen challenges.
These results complement the hygiene hypothesis, which states that although high levels of microbial diversity in the environment are associated with a reduced risk of asthma, the colonization of specific dominant bacteria, such as NTHi, in the bronchi may be a risk factor for asthma.

\section{NTHi infection and corticosteroid-resistant asthma}

At present, the symptoms of most asthmatic patients can be well controlled by treatment with inhaled corticosteroids combined with long-acting beta agonists, but some patients have a poor response to corticosteroids therapy; high-dose corticosteroids therapy is difficult to control, and the condition of repeated acute aggravation is called steroid resistance. Corticosteroid-resistant asthma is a serious global problem, leading to an enormous economic burden, and is one of the main causes of death in asthmatic patients. At the same time, corticosteroid resistance in asthma is a very complex problem. Current research shows that the mechanism of corticosteroid resistance may be related to genetic variation, epigenetics, asthma phenotypes, race and the airway microbiome [60].

NTHi is one of the most common bacteria isolated from asthmatic patients and is also associated with neutrophilic asthma and corticosteroid resistance in asthma. In vitro, Haemophilus influenzae has been shown to inhibit the corticosteroid response in asthmatic alveolar macrophages and peripheral blood mononuclear cells [61]. After NTHi infection, the TGF- $\beta$-activated kinase (TAK)-1/MAPK pathway was activated, and corticosteroid resistance was induced. Inhibition of the TAK-1 pathway can restore the sensitivity of cells to corticosteroids, suggesting that the development of corticosteroid resistance may be related to the TAK-1 pathway [62].

Another important mechanism of NTHi infection that promotes corticosteroid resistance is that NTHi infection leads to Th17/Treg homeostasis imbalances (as described in section 3.4), which leads to the occurrence of a neutrophilic asthma phenotype. IL-17 increased significantly after NTHi infection, which may be an important reason that NTHi infection promoted corticosteroid resistance. The transplantation of ova-treated Th17 cells into severely immunodeficient mice caused severe corticosteroid-resistant asthma [63], which confirmed the role of Th17 cells in corticosteroid resistance. IL-17 can counteract the effects of corticosteroids in human airway epithelial cells by inducing epigenetic changes and maintaining the secretion of inflammatory factors. It has been proven that IL-17 can affect the binding of glucocorticoid receptor (GR) to glucocorticoids (GS) by activating the phosphoinositide-3-kinase (PI3K) pathway and reducing histone deacetylase 2 (HDAC2) activity [64]. In addition, it was observed that IL-17 could 
increase the expression of GRb [65] in the peripheral blood cells of healthy donors. In patients with corticosteroid resistance, the imbalance of GRa/GRb may affect the binding of GR to CS, which may also lead to the occurrence of corticosteroid resistance.

In addition, another mechanism of NTHi colonization that promotes corticosteroid resistance may be related to oxidative stress. NTHi activates oxidative stress strongly after airway infection, as described in section 3.2 , which causes airway smooth muscle contraction, the induction of airway hyperresponsiveness, excessive mucus secretion, epithelial exfoliation, vascular exudation and airway remodeling $[25,38]$. Oxidative stress can also lead to further inflammation and damage the airway mucosa, promoting continuous colonization of NTHi and the invasion of other pathogens, which aggravate airway microbial disorders. Furthermore, oxidative stress can impair GR activity by lowering the activity of HDAC2, which has been shown to promote corticosteroid resistance in the lungs of patients with corticosteroid-resistant asthma $[66,67]$.

\section{Treatment of NTHi-related neutrophilic asthma}

Presently, a large number of new studies on asthma treatment have emerged, providing many new ideas for clinical treatments. In particular, NTHi may be associated with the aggravation of neutrophilic asthma, so the targeted treatment of NTHi may effectively improve the symptoms of neutrophilic asthma.

\section{Use of antibiotics}

Using antibiotics to treat NTHi colonization is a direct and effective method, and macrolides are more suitable than other antibiotics. Studies have shown that longterm, low-dose azithromycin can regulate airway inflammation and inhibit NTHi-induced MUC5AC synthesis at the mRNA and protein levels by selectively inhibiting the activation of transcription factor activator protein-1 [68]. However, the long-term use of antibiotics results in the emergence of drug-resistant strains [12]. At the same time, an increasing number of studies have shown that children and pregnant women exposed to antibiotics are at increased risk of asthma later in life or in their newborn babies, and this risk is positively correlated with an increased antimicrobial spectrum and an increased antibiotic dosage [69-72]. The GINA report does not support the use of antibiotics for asthma, and it is recommended that antibiotics should not be routinely used to treat exacerbations of asthma unless there is definitive evidence of pulmonary infection (e.g., radiological evidence of pneumonia, fever or suppurative sputum) [2].

Therefore, when asthma is accompanied by NTHi infection, it is recommended to weigh the advantages and disadvantages and to decide whether to use antibiotics. The newly synthesized nonantibiotic macrolides have shown promise as prospective agents. These compounds can not only significantly improve macrophage phagocytosis, which is disturbed by NTHi, but can also regulate airway inflammation. At the same time, the antimicrobial activity of the newly synthesized nonantibiotic macrolides is significantly lower than that of antibiotics, thus significantly reducing the emergence of drug-resistant bacteria [73].

\section{Other anti-inflammatory drugs}

In addition to antibiotics, recent studies have found that some drugs targeting NTHi also have anti-inflammatory effects. Curcumin (from Curcuma plants) has a strong anti-inflammatory ability in otitis media caused by NTHi infection, and it can inhibit the NTHi-mediated upregulation of C-X-C motif chemokine 5 (CXCL5) expression by activating the inhibitor of nuclear factor kappa-B kinase kinase- $\beta$ (IKK $\beta)$-inhibitor kappa $B(\mathrm{I} \kappa \mathrm{B} \alpha)$ and p38 MAPK pathways [74]. Studies have also shown that curcumin can inhibit airway inflammation and MUC5AC secretion in asthmatic mice [75]. Whether curcumin is effective in NTHi-induced neutrophilic asthma needs further study.

Resveratrol has long been considered an interesting drug for the treatment of various inflammatory diseases. Studies on its anti-inflammatory mechanism suggest that resveratrol increases the expression of MAPK phosphatase-1 through the camp/pka-dependent signaling pathway and inhibits NTHi-induced ERK1/2 phosphorylation. These results suggest that resveratrol has an anti-inflammatory effect after NTHi infection, so it has therapeutic potential in NTHi-related neutrophilic asthma.

Vitamin D is a unique nutrient that is multipotent, and it is believed to play a very important role in immune regulation. In addition, there is growing evidence that vitamin $\mathrm{D}$ also plays a protective role in lung diseases, such as allergic airway diseases, lung cancer and pulmonary fibrosis [76-78]. Vitamin D deficiency can increase the incidence of asthma and allergic airway diseases, and lung function can be improved by vitamin D supplementation, especially in patients with vitamin D deficiency [79]. Recent animal experiments have shown that $1,25-(\mathrm{OH}) 2 \mathrm{D} 3$ can regulate IL-17A at the transcriptional level through Runx1, thus alleviating airway inflammation in mice with neutrophilic asthma [80]. At the same time, it was observed in clinical trials that vitamin D can significantly improve steroid resistance in patients with severe asthma [81]. Therefore, it is speculated that vitamin $\mathrm{D}$ has potential value in the treatment of NTHi-related neutrophilic asthma. 


\section{Vaccines against NTHi}

Epidemiological studies have shown that an increase in NTHi infection occurs after widespread vaccination with the Haemophilus influenzae type b conjugate vaccine [82]. Because NTHi can survive in cells and has many mechanisms to escape host immunity, routine therapy cannot eradicate NTHi colonization, and recurrent infection and chronic airway inflammation are common. Therefore, developing an NTHi vaccine may be key to preventing and treating NTHi-related diseases in the future [83].

\section{Targeted therapy for IL-17}

The importance of Th17/Treg homeostasis in the pathogenesis of neutrophilic asthma has been recognized by researchers. The NTHi-induced aggravation of neutrophilic asthma is closely related to an increase in IL-17. Interventions targeting Th17/Treg cells may be a new method for treating NTHi-related neutrophilic asthma, especially corticosteroid-resistant neutrophilic asthma $[48,49]$. At present, many drugs targeting IL-17 are in clinical studies [84-86]. An antibody that neutralizes the human receptor for IL-17 (brodalumab, which can neutralize the activities of IL-17A, IL-17F and IL-25) has little effect on patients with mild to moderate asthma in clinical trials and has not achieved satisfactory results [87]. This may be related to the complex pathogenesis of neutrophilic asthma. In addition to Th17 cells and Treg cells, many immunoregulatory cells, such as Th2 cells, Th1 cells, innate lymphoid cells, dendritic cells, natural killer T cells, and TH9 cells, and many endogenous cytokines related to asthma, such as IL4, IL-5, IL13, IL22, and IL25, are also involved [1].

Neutrophilic asthma is regarded as a disease that has an exclusive Th17/Treg imbalance, which may be too simplified and seen only in extreme cases. In the clinic, various types of endogenous cytokines may overlap considerably and may be related to the severity of asthma. Therefore, more research and clinical work are needed on IL-17 targeted therapy.

\section{Immunotherapy with oral NTHi}

Earlier studies have found that sputum culture positivity and acute attack frequency were significantly rescued in moderate to severe COPD patients after the oral inactivation of NTHi [88]. Further studies have found that one of the mechanisms underlying the exacerbation of COPD is bacterial colonization, especially NTHi colonization, which results in neutrophilic inflammation in damaged airways, and this inflammation promotes the acute exacerbation of COPD [89]. In light of this hypothesis, oral immunotherapy with NTHi may enhance the delivery of intestinal antigens to Peyer's patches, increase the number of $\mathrm{T}$ cells transported to the airway mucosa, and improve the efficiency of bacterial phagocytosis [89]. The protective effect of this treatment involves reducing the amount of NTHi in the damaged bronchi and protecting the airway. Based on this hypothesis, oral NTHi immunotherapy may also play a role in the aggravation of NTHi-induced neutrophilic asthma. To date, there have been no clinical studies on oral NTHi immunotherapy for neutrophilic asthma, which could be a good starting point for the treatment of neutrophilic asthma.

\section{Nrf2 activators and antioxidants}

Oxidative stress plays an essential role in asthma-related neutrophilic inflammation, AHR, increased mucus secretion and airway remodeling. It has been reported that asthmatic patients may have more difficulty coping with oxidative stress than healthy people, which may be closely related to the impairment of Nrf2 activity. Recent studies have also shown that Nrf2-driven glutathione-Stransferase may be a marker of susceptibility to asthma in humans [90]. Nrf2 agonists such as sulforaphane can inhibit neutrophilic airway inflammation and improve asthma symptoms in a mouse asthma model. Some classical antioxidants, such as vitamin $\mathrm{E}$, can improve asthma symptoms by activating the $\mathrm{Nrf} 2$ /heme oxygenase (HO)-1 pathway [91]. Combined with the current achievements in animal experiments, Nrf2 agonists and antioxidants may have great potential for application in NTHi-mediated neutrophilic asthma, but more evidence from different populations is needed for the clinical application of these treatments.

\section{Development potential and clinical applications of neutrophilic asthma drugs}

The research and development of drugs for asthma and neutrophilic asthma are ongoing. The most recent clinical trial of asthma treatment is a phase IB clinical trial (EQUIP) of itolizumab. Itolizumab is a CD6-targeted antibody that has been suggested for the treatment of autoimmune diseases (such as psoriasis and arthritis). To date, the EQUIP clinical trial has not been completed, but itolizumab has been found to inhibit different effector T cell subtypes (including Th2 and Th17 cells), which play important roles in the pathogenesis of asthma; therefore, itolizumab may play a role in neutrophilic asthma [92].

The proinflammatory cytokine thymic stromal lymphopoietin (TSLP) is an epithelial cytokine produced by proinflammatory stimulation (such as that caused by pulmonary allergens, viruses and other pathogens) that plays a key role in the occurrence and persistence of airway inflammation. TSLP drives the release of downstream Th2 cytokines (including IL-4, IL-5 and IL-13) and activates various types of cells involved in non-Th2-driven 
inflammation. Tezepelumab is an anti-TSLP monoclonal antibody that specifically binds to human TSLP to block its interaction with its receptor complex. Tezepelumab is currently not listed in any country. In September 2017, AstraZeneca, a British pharmaceutical company, and Amgen, an AstraZeneca partner, published positive results of a phase $2 \mathrm{~B}$ clinical trial conducted on tezepelumab use in patients with uncontrolled asthma, which showed that compared with that of patients taking a placebo, the annual asthma attack rate of patients receiving tezepelumab decreased by $71 \%$. This result does not reflect the baseline eosinophil count and indicates the great potential of tezepelumab in the treatment of neutrophilic asthma [93, 94]. In October 2018, AstraZeneca and Amgen jointly announced that the US Food and Drug Administration (FDA) granted tezepelumab a breakthrough drug designation (BTD) for the treatment of patients with severe asthma with a noneosinophilic phenotype. Currently, tezepelumab is in phase III clinical development.

Neutrophilic asthma is usually characterized by corticosteroid resistance and severity; that is, the effect of corticosteroid treatment is unsatisfactory. The latest research shows that the new high-efficiency glucocorticoid ligands (such as vsg158) had a very high antiinflammatory effect in animal asthma models, showing an effect 10-fold stronger than that of the widely used and effective asthma drug (fluticasone furoate, FF) and reversed corticosteroid resistance in the animal model of a corticosteroid-resistant type of malignant asthma. Although no clinical experiments were carried out, the research provided a new idea for the treatment of malignant asthma characterized by high mortality [95].

Neutrophils express chemokine receptors 1 and 2 (CXCR1 and 2) on the cell surface, interact with chemokines (such as interleukin 8), and recruit neutrophils to the site of inflammation or injury. Therefore, it has been suggested that the neutrophil chemokine receptor may be a potential therapeutic target for neutrophilic asthma. Azd5069 is a CXCR2 chemokine receptor antagonist that blocks the effect of interleukin 8. In a 6-month phase 2B clinical study on azd5069, although compared to the placebo, azd5069 reduced the mean blood neutrophil count, neither significant differences in the frequency of asthma attacks or symptoms nor changes in lung function were found. The definition of the neutrophilic asthma phenotype in this study was based on the fact that the blood neutrophil count was increased and the blood eosinophil count was reduced 0.5 -fold. At $10^{9} / \mathrm{L}$, the threshold eosinophil count may have been too high, resulting in some patients with an eosinophilic asthma subtype (combined with neutrophil elevation) being included in the trial, resulting in unsatisfactory treatment results. This outcome suggests that the blood neutrophil count may not be a good marker of neutrophilic asthma [96].
Recent research and development of drugs for neutrophilic asthma and related clinical experiments have provided some potential targets for the treatment of neutrophilic asthma, with some gratifying results. With continued research and an increased understanding of the potential mechanism of neutrophilic inflammation, it is imperative to use different drugs for different asthma subtypes, and more and larger clinical experiments are needed to promote progress in drug research.

\section{Conclusion}

Neutrophilic asthma is a subtype of asthma that is closely related to the corticosteroid resistance of asthma and causes an enormous medical burden. The mechanism of airway inflammation in neutrophilic asthma is very complex; currently, its pathogenesis is not completely clear. Biological disorders of the lower airway have been shown to contribute to the worsening of neutrophilic asthma. NTHi is one of the most common bacteria in lower respiratory tract flora disorders; it is becoming increasingly clear that NTHi plays an important role in the progression of neutrophilic asthma and promotes the occurrence of corticosteroid resistance. In this study, the mechanisms of NTHi that promote the worsening of neutrophilic asthma were analyzed to explore the possible regulatory mechanisms in neutrophilic asthma, including hypersecretion of mucus, oxidative stress, Th1/Th2 imbalance and Th17/Treg imbalance. However, we should also note that these studies are incomplete, and many issues remain poorly understood, such as the study of how Th17 cells and Th2 cells cooperate in neutrophilic asthma and how NTHi infection cooperates with or antagonizes other bacterial and viral infections in the lower airway disorders of neutrophilic asthma; these topics deserve further study.

In view of the role of NTHi in neutrophilic asthma, many therapeutic measures against NTHi in asthmatic patients are being explored at present, such as the use of antibiotics, the use of other anti-inflammatory drugs, the development of an NTHi vaccine, IL-17 targeted therapy, oral NTHi immunotherapy, and antioxidants for the increased airway oxidative stress caused by NTHi. Clinical trials for drug research and development for neutrophilic asthma are also in progress, such as trials of itolizumab and tezepelumab, and some satisfactory results have been obtained, showing the potential of targeted treatment for neutrophilic asthma.

In conclusion, NTHi infection provides a new target for studying the pathogenesis and treatment of neutrophilic asthma. It is believed that with additional research, the pathogenesis of neutrophilic asthma will be further elucidated, and more targeted drugs will be designed for the specific phenotype and endotype of neutrophilic asthma. 


\section{Abbreviations}

ICS: inhaled corticosteroids; GINA: Global Initiative for Asthma; IL: interleukin; AHR: airway hyperreactivity; NTHi: Nontypeable Haemophilus influenzae; LOSs: lipooligosaccharides; PAFR: platelet activating factor receptor; COPD: chronic obstructive pulmonary disease; MAPK: mitogen-activated protein kinase; NF-kB: nuclear factor-kappa B; ROS: oxygen species; Nrf2: Nuclear factor erythroid 2-related factor 2; NETs: neutrophil extracellular traps; ASM: airway smooth muscle; OVA: ovalbumin; AADs: allergic airway diseases; TAK: TGF- $\beta$-activated kinase; GR: Glucocorticoid receptor; GS: Glucocorticoids; PI3K: Phosphoinositide-3-kinase; HDAC2: histone deacetylase 2; CXCL5: C-X-C motif chemokine 5; IKKB: inhibitor of nuclear factor kappa-B kinase- $\beta$; IkBa: inhibitor kappa B; HO: heme oxygenase; TSLP: thymic stromal lymphopoietin;

\section{Acknowledgments}

Not applicable.

\section{Compliance with ethical standards}

Not applicable.

\section{Informed consent}

Not applicable.

\section{Authors' contributions}

Conceptualization: JZ, ZXZ and XZ. Literature search and data analysis: HP, YNG, YY and RPZ. Writing - original draft: JZ, GQW and SJW. Writing reviewing and editing: $Z M L, F W$ and JTZ. The authors read and approved the final manuscript.

\section{Funding}

This work was supported by the Jilin Health Committee (2017J026) and Jilin Province Science and Technology Development Plan Project (20170623093-07TC).

\section{Availability of data and materials}

All data generated or analyzed during this study are included in this published article.

\section{Ethics approval and consent to participate}

Not applicable.

\section{Consent for publication}

Not applicable.

\section{Competing interests}

The authors declare that they have no competing interests.

\section{Author details}

${ }^{1}$ Department of Intensive Care Unit, First Hospital of Jilin University, Changchun 130021, China. 'Department of Pathogen Biology, College of Basic Medical Sciences, Jilin University, Changchun 130021, China. ${ }^{3}$ Department of Hematology and Oncology, China-Japan Union Hospital of Jilin University, Changchun 130033, China. ${ }^{4}$ Department of Interventional Therapy, First Hospital of Jilin University, Changchun 130021, China. ${ }^{5}$ Key Laboratory of Zoonosis, Ministry of Education, College of Veterinary Medicine, Jilin University, Changchun 130062, China.

Received: 20 December 2019 Accepted: 30 June 2020

Published online: 03 July 2020

\section{References}

1. Lambrecht BN, Hammad H. The immunology of asthma. Nat Immunol. 2015;16:45-56

2. Reddel HK, Bateman ED, Allan B, Louis-Philippe B, Cruz AA, Drazen JM, Tari H, Hurd SS, Hiromasa I, de Jongste Johan C, Lemanske RF, Levy ML, O'Byrne PM, Pierluigi P, Pedersen SE, Emilio P, Manuel S-Q, Szefler SJ, Wong Gary WK, Mark FGJ. A summary of the new GINA strategy: a roadmap to asthma control. Eur Respir J. 2015;46:622-39.

3. Simpson JL, Scott R, Boyle MJ, Gibson PG. Inflammatory subtypes in asthma: assessment and identification using induced sputum. Respirology. 2006;11:54-61.
4. McCann JR, Mason SN, Auten RL, St. Geme JW, Seed PC. Early-life intranasal colonization with nontypeable Haemophilus influenzae exacerbates juvenile airway disease in mice. Infect Immun. 2016;84:2022-30.

5. Peters, Michael C., Sheena Kerr, Eleanor M. Dunican, Prescott G. Woodruff, Merritt L. Fajt, Bruce D. Levy, Elliot Israel, Brenda R. Phillips, David T. Mauger, Suzy A. Comhair, Serpil C. Erzurum, Mats W. Johansson, Nizar N. Jarjour, Andrea M. Coverstone, Mario Castro, Annette T. Hastie, Eugene R. Bleecker, Sally E. Wenzel, and John V. Fahy. 2019. Refractory airway type 2

inflammation in a large subgroup of asthmatic patients treated with inhaled corticosteroids. J Allergy Clin Immunol 143: 104-113.e114.

6. Hansbro PM, Kim RY, Starkey MR, Donovan C, Dua K, Mayall JR, Liu G, Hansbro NG, Simpson JL, Wood LG, Hirota JA, Knight DA, Foster PS, Horvat JC. Mechanisms and treatments for severe, steroid-resistant allergic airway disease and asthma. Immunol Rev. 2017;278:41-62.

7. Liu AH. Revisiting the hygiene hypothesis for allergy and asthma. J Allergy Clin Immunol. 2015;136:860-5.

8. Bonamichi-Santos R, Aun MV, Agondi RC, Kalil J, Giavina-Bianchi P. Microbiome and asthma: what have experimental models already taught us? J Immunol Res. 2015;2015:614758.

9. Lu J, Xiong L, Zhang X, Liu Z, Wang S, Zhang C, Zheng J, Wang G, Zheng R, Simpson JL, Wang F. The role of lower airway dysbiosis in asthma: Dysbiosis and asthma. Mediat Inflamm. 2017;2017:1-10.

10. Hilty M, Burke C, Pedro H, Cardenas P, Bush A, Bossley C, Davies J, Ervine A, Poulter L, Pachter L, Moffatt MF, Cookson WOC. Disordered microbial communities in asthmatic airways. PLoS One. 2010;5:e8578.

11. Simpson JL, Daly J, Baines KJ, Yang IA, Upham JW, Reynolds PN, Hodge S, James AL, Hugenholtz P, Willner D, Gibson PG. Airway dysbiosis: Haemophilus influenzae and Tropheryma in poorly controlled asthma. Eur Respir J. 2015:47:792-800.

12. Van Eldere J, Slack MPE, Ladhani S, Cripps AW. Non-typeable Haemophilus influenzae, an under-recognised pathogen. Lancet Infect Dis. 2014;14:1281-92.

13. Gessner BD, Adegbola RA. The impact of vaccines on pneumonia: key lessons from Haemophilus influenzae type b conjugate vaccines. Vaccine. 2008;26:B3-8.

14. Taylor SL, Leong LEX, Choo JM, Wesselingh S, Yang IA, Upham JW, Reynolds PN, Hodge S, James AL, Jenkins C, Peters MJ, Baraket M, Marks GB, Gibson PG, Simpson JL, Rogers GB. Inflammatory phenotypes in patients with severe asthma are associated with distinct airway microbiology. J Allergy Clin Immunol. 2018;141:94-103 e115.

15. Yang, Xu, Yunqiu Jiang, and Changzheng Wang. 2016. Does IL-17 respond to the disordered lung microbiome and contribute to the neutrophilic phenotype in asthma? Mediat Inflamm 2016: 1-7.

16. Allen S, Zaleski A, Johnston JW, Gibson BW, Apicella MA. Novel sialic acid transporter of Haemophilus influenzae. Infect Immun. 2005;73:5291-300.

17. Toropainen M, Raitolehto A, Henckaerts I, Wauters D, Poolman J, Lestrate P, Käyhty H. Pneumococcal Haemophilus influenzae protein D conjugate vaccine induces antibodies that inhibit glycerophosphodiester phosphodiesterase activity of protein D. Infect Immun. 2008;76:4546-53.

18. Kenjale R, Meng G, Fink DL, Juehne T, Ohashi T, Erickson HP, Waksman G, St Geme JW. Structural determinants of autoproteolysis of the Haemophilus influenzae hap autotransporter. Infect Immun. 2009;77:4704-13.

19. Murphy TF, Lesse AJ, Kirkham C, Zhong H, Sethi S, Munson RS. A clonal group of nontypeable Haemophilus influenzae with two IgA proteases is adapted to infection in chronic obstructive pulmonary disease. PLoS One. 2011;6:e25923.

20. Murphy TF, Kirkham C, Jones MM, Sethi S, Kong Y, Pettigrew MM. Expression of IgA proteases by Haemophilus influenzae in the respiratory tract of adults with chronic obstructive pulmonary disease. J Infect Dis. 2015;212:1798-805.

21. Bandi V, Apicella MA, Mason E, Murphy TF, Siddiqi A, Atmar RL, Greenberg SB. Nontypeable Haemophilus influenzae in the lower respiratory tract of patients with chronic bronchitis. Am J Respir Crit Care Med. 2001;164:2114-9.

22. Weiser JN, Pan N. Adaptation of Haemophilus influenzae to acquired and innate humoral immunity based on phase variation of lipopolysaccharide. Mol Microbiol. 1998;30:767-75.

23. Clementi CF, Håkansson AP, Murphy TF. Internalization and trafficking of nontypeable Haemophilus influenzae in human respiratory epithelial cells and roles of lgA1 proteases for optimal invasion and persistence. Infect Immun. 2014;82:433-44. 
24. Shukla SD, Fairbairn RL, Gell DA, Latham R, Sohal SS, Walters H, OToole R. An antagonist of the platelet-activating factor receptor inhibits adherence of both nontypeable Haemophilus influenzae and Streptococcus pneumoniae to cultured human bronchial epithelial cells exposed to cigarette smoke. Int J Chronic Obstructive Pulmonary Disease. 2016;11:1647-55.

25. Clementi CF, Murphy TF. Non-typeable Haemophilus influenzae invasion and persistence in the human respiratory tract. Front Cell Infect Microbiol. 2011; $1: 1$.

26. King PT, Sharma R. The lung immune response to nontypeable Haemophilus influenzae (lung immunity to NTHi). J Immunol Res. 2015;2015:706376.

27. Essilfie A-T, Simpson JL, Horvat JC, Preston JA, Dunkley ML, Foster PS, Gibson PG, Hansbro PM. Haemophilus influenzae infection drives IL-17mediated neutrophilic allergic airways disease. PLoS Pathog. 2011;7: e1002244.

28. Jurcisek JA, Brockman KL, Novotny LA, Goodman SD, Bakaletz LO. Nontypeable haemophilus influenzae releases DNA and DNABII proteins via a T4SS -like complex and ComE of the type IV pilus machinery. Proc Natl Acad Sci. 2017;114:201705508.

29. Wang B, Lim DJ, Han J, Kim YS, Basbaum CB, Li J-D. Novel cytoplasmic proteins of nontypeable Haemophilus influenzae up-regulate human MUC5AC mucin transcription via a positive p38 mitogen-activated protein kinase pathway and a negative phosphoinositide 3-kinase-Akt pathway. J Biol Chem. 2002;277:949-57.

30. McNamara N, Gallup M, Khong A, Sucher A, Maltseva I, Fahy J, Basbaum C. Adenosine up-regulation of the mucin gene, MUC2, in asthma. FASEB J. 2004:18:1770-2.

31. Jono H, Shuto T, Xu H, Kai H, Lim DJ, Gum JR, Kim YS, Yamaoka S, Feng X-H, Li J-D. Transforming growth factor- $\beta$-smad signaling pathway cooperates with NF-KB to mediate nontypeable Haemophilus influenzae-induced MUC2 mucin transcription. J Biol Chem. 2002;277:45547-57.

32. Andreadis, Athena A., Stanley L. Hazen, Suzy A. A. Comhair, and Serpil C. Erzurum. 2003. Oxidative and nitrosative events in asthma. Free Radic Biol Med 35: 213-225.

33. Rangasamy T, Guo J, Mitzner WA, Roman J, Singh A, Fryer AD, Yamamoto M, Kensler TW, Tuder RM, Georas SN, Biswal S. Disruption of Nrf2 enhances susceptibility to severe airway inflammation and asthma in mice. J Exp Med. 2005;202:47-59

34. Nadeem A, Siddiqui N, Al-Harbi NO, Al-Harbi MM, Ahmad SF. TLR-7 agonist attenuates airway reactivity and inflammation through Nrf2-mediated antioxidant protection in a murine model of allergic asthma. Int J Biochem Cell Biol. 2016:73:53-62.

35. Liu Q, Gao Y, Ci X. Role of Nrf2 and its activators in respiratory diseases. Oxidative Med Cell Longev. 2019;2019:1-17.

36. King PT, Sharma R, O'Sullivan K, Selemidis S, Lim S, Radhakrishna N, Lo C, Prasad J, Callaghan J, McLaughlin P, Farmer M, Steinfort D, Jennings B, Ngui J, Broughton BRS, Thomas B, Essilfie A-T, Hickey M, Holmes PW, Hansbro P, Bardin PG, Holdsworth SR. Nontypeable Haemophilus influenzae induces sustained lung oxidative stress and protease expression. PLoS One. 2015;10: e0120371.

37. Harrison A, Baker BD, Munson RS. Overlapping and complementary oxidative stress defense mechanisms in nontypeable Haemophilus influenzae. J Bacteriol. 2015;197:277-85.

38. Harrison A, Bakaletz LO, Munson RS. Haemophilus influenzae and oxidative stress. Front Cell Infect Microbiol. 2012;2:40.

39. Wong $\mathrm{C}$, Jones $\mathrm{S}$. Oxidative stress and macrolides in bronchiectasis exhaling few clues. Respirology. 2013;18:1037-8.

40. Cho YS, Moon H-B. The role of oxidative stress in the pathogenesis of asthma. Allergy Asthma Immunol Res. 2010;2:183-7.

41. Hardy GG, Tudor SM, Geme JWS. The pathogenesis of disease due to nontypeable Haemophilus influenzae. Methods Molecular Med. 2003;71:1-28.

42. Kumari A, Singh DK, Dash D, Singh R. Intranasal curcumin protects against LPS-induced airway remodeling by modulating toll-like receptor-4 (TLR-4) and matrixmetalloproteinase-9 (MMP-9) expression via affecting MAP kinases in mouse model. Inflammopharmacology. 2018;27:731-48.

43. Comhair SAA, Erzurum SC. Redox control of asthma: molecular mechanisms and therapeutic opportunities. Antioxid Redox Signal. 2010;12:93-124.

44. Reddel HK, Levy ML. The GINA asthma strategy report: What's new for primary care? NPJ Primary Care Respiratory Medicine. 2015;25:15050.

45. Won J, Gil CH, Jo A, Kim HJ. Inhaled delivery of interferon-lambda restricts epithelial-derived Th2 inflammation in allergic asthma. Cytokine. 2019;119: $32-6$.
46. Wenzel SE. Asthma phenotypes: the evolution from clinical to molecular approaches. Nat Med. 2012;18:716-25.

47. Berker M, Frank LJ, Geßner AL, Grassl N, Holtermann AV, Höppner S, Kraef C, Leclaire MD, Maier P, Messerer DAC, Möhrmann L, Nieke JP, Schoch D, Soll D, Woopen CMP. Allergies - a T cells perspective in the era beyond the TH1/TH2 paradigm. Clin Immunol. 2017;174:73-83.

48. Hirose K, Iwata A, Tamachi T, Nakajima H. Allergic airway inflammation: key players beyond the Th2 cell pathway. Immunol Rev. 2017;278:145-61.

49. Wilson RH, Whitehead GS, Nakano H, Free ME, Kolls JK, Cook DN. Allergic sensitization through the airway primes Th17-dependent neutrophilia and airway hyperresponsiveness. Am J Respir Crit Care Med. 2009;180:720-30.

50. Zhao J, Lloyd CM, Noble A. Th17 responses in chronic allergic airway inflammation abrogate regulatory T-cell-mediated tolerance and contribute to airway remodeling. Mucosal Immunol. 2013;6:335-46.

51. Guan $X$, Lu Y, Wang G, Gibson $P$, Chen F, Fang K, Wang Z, Pang Z, Guo Y, Lu J, Yuan Y, Ran N, Wang F. The role of regulatory T cell in nontypeable Haemophilus influenzae-induced acute exacerbation of chronic obstructive pulmonary disease. Mediat Inflamm. 2018;2018:1-14.

52. Zhu L, Ciaccio CE, Casale TB. Potential new targets for drug development in severe asthma. World Allergy Organization Journal. 2018;11:30.

53. Webley WC, Hahn DL. Infection-mediated asthma: etiology, mechanisms and treatment options, with focus on Chlamydia pneumoniae and macrolides. Respir Res. 2017;18:98.

54. Bellini A, Marini MA, Bianchetti L, Barczyk M, Schmidt ME, Mattoli S. Interleukin (IL)-4, IL-13, and IL-17A differentially affect the profibrotic and proinflammatory functions of fibrocytes from asthmatic patients. Mucosal Immunol. 2012;5:140-9.

55. Tan H-TT, Hagner S, Ruchti F, Radzikowska U, Tan G, Altunbulakli C, Eljaszewicz A, Moniuszko M, Akdis M, Akdis CA, Garn H, Sokolowska M. Tight junction, mucin, and inflammasome-related molecules are differentially expressed in eosinophilic, mixed, and neutrophilic experimental asthma in mice. Allergy. 2018;74:294-307.

56. Al-Alwan LA, Chang Y, Baglole CJ, Risse P-A, Halayko AJ, Martin JG, Eidelman $\mathrm{DH}$, Hamid Q. Autocrine-regulated airway smooth muscle cell migration is dependent on IL-17-induced growth-related oncogenes. J Allergy Clin Immunol. 2012;130:977-85 e976.

57. Chang Y, Al-Alwan L, Risse P-A, Halayko AJ, Martin JG, Baglole CJ, Eidelman DH, Hamid Q. Th17-associated cytokines promote human airway smooth muscle cell proliferation. FASEB J. 2012;26:5152-60.

58. Loubaki L, Hadj-Salem I, Fakhfakh R, Jacques E, Plante S, Boisvert M, Aoudjit F, Chakir J. Co-culture of human bronchial fibroblasts and CD4+ T cells increases Th17 cytokine signature. PLoS One. 2013:8:e81983.

59. Wang F, He XY, Baines KJ, Gunawardhana LP, Simpson JL, Li F, Gibson PG. Different inflammatory phenotypes in adults and children with acute asthma. Eur Respir J. 2011;38:567-74.

60. Ramadan AA, Gaffin JM, Israel E, Phipatanakul W. Asthma and corticosteroid responses in childhood and adult asthma. Clin Chest Med. 2019:40:163-77.

61. Goleva E, Harris JK, Robertson CE, Jackson LP, Martin RJ, Leung DYM. Airway microbiome and responses to corticosteroids in corticosteroid-resistant asthma patients treated with acid suppression medications. J Allergy Clin Immunol. 2017;140:860-2 e861.

62. Goleva E, Jackson LP, Harris JK, Robertson CE, Sutherland ER, Hall CF, Good JT, Gelfand EW, Martin RJ, Leung DYM. The effects of airway microbiome on corticosteroid responsiveness in asthma. Am J Respir Crit Care Med. 2013; 188:1193-201.

63. McKinley L, Alcorn JF, Peterson A, DuPont RB, Kapadia S, Logar A, Henry A, Irvin CG, Piganelli JD, Ray A, Kolls JK. TH17 cells mediate steroid-resistant airway inflammation and airway hyperresponsiveness in mice. J Immunol. 2008;181:4089-97.

64. Zijlstra GJ, ten Hacken N, Hoffmann R, van Oosterhout AJM, Heijink IH. Interleukin-17A induces glucocorticoid insensitivity in human bronchial epithelial cells. Eur Respir J. 2012;39:439-45.

65. Vazquez-Tello A, Semlali A, Jamila C, Martin JG, Dexter L, Eidelman DH, Hamid Q. Induction of glucocorticoid receptor- $\beta$ expression in epithelial cells of asthmatic airways by T-helper type 17 cytokines. Clin Exp Allergy. 2010:40:1312-22.

66. Bhavsar PK, Hew M, Nematolla K, Torrego A, Barnes PJ, Adcock IM, Chung KF. Relative corticosteroid insensitivity of alveolar macrophages in severe asthma compared with non-severe asthma. Thorax. 2008;63:784-90.

67. Hew M, Bhavsar P, Torrego A, Meah S, Khorasani N, Barnes PJ, Adcock I, Chung KF. Relative corticosteroid insensitivity of peripheral blood 
mononuclear cells in severe asthma. Am J Respir Crit Care Med. 2006;174: 134-41.

68. Araki N, Yanagihara K, Morinaga Y, Yamada K, Nakamura S, Yamada Y, Kohno S, Kamihira S. Azithromycin inhibits nontypeable Haemophilus influenzae-induced MUC5AC expression and secretion via inhibition of activator protein-1 in human airway epithelial cells. Eur J Pharmacol. 2010;644:209-14.

69. Semic-Jusufagic A, Belgrave D, Pickles A, Telcian AG, Bakhsoliani E, Sykes A, Simpson A, Johnston SL, Custovic A. Assessing the association of early life antibiotic prescription with asthma exacerbations, impaired antiviral immunity, and genetic variants in 17q21: a population-based birth cohort study. Lancet Respir Med. 2014;2:621-30.

70. Chen IL, Huang H-C, Chang Y-H, Huang H-Y, Yeh W-J, Wu T-Y, Suen J-L, Yang S-N, Hung C-H. Effect of antibiotic use for acute bronchiolitis on newonset asthma in children. Sci Rep. 2018;8:6090.

71. Donovan BM, Abreo A, Tan D, Gebretsadik T, Turi KN, Yu C, Ding J, Dupont WD, Stone CA, Hartert TV, Pingsheng W. Dose, timing, and type of infant antibiotic use and the risk of childhood asthma. Clin Infect Dis. 2019. https://doi.org/10.1093/cid/ciz448.

72. Loewen K, Monchka B, Mahmud SM, Jong GT, Azad MB. Prenatal antibiotic exposure and childhood asthma: a population-based study. Eur Respir J. 2018;52:1702070.

73. Hodge S, Tran HB, Hamon R, Roscioli E, Hodge G, Jersmann H, Ween M, Reynolds PN, Yeung A, Treiberg J, Wilbert S. Nonantibiotic macrolides restore airway macrophage phagocytic function with potential antiinflammatory effects in chronic lung diseases. Am J Phys Lung Cell Mol Phys. 2017;312:L678-87.

74. Konduru AS, Lee B-C, Li J-D. Curcumin suppresses NTHi-induced CXCL5 expression via inhibition of positive IKK $\beta$ pathway and up-regulation of negative MKP-1 pathway. Sci Rep. 2016;6:31695.

75. Zhu T, Chen Z, Chen G, Wang D, Tang S, Deng H, Wang J, Li S, Lan J, Tong J, He L, Deng X, Zhang W, Sun J, Yuesheng T, Luo W, Li C. Curcumin attenuates asthmatic airway inflammation and mucus hypersecretion involving a PPARY-dependent NF-KB signaling pathway in vivo and in vitro. Mediat Inflamm. 2019;2019:4927430

76. Akiba T, Morikawa T, Odaka M, Nakada T, Kamiya N, Yamashita M, Yabe M, Inagaki T, Asano H, Mori S, Tsukamoto Y, Urashima M. Vitamin D supplementation and survival of patients with non-small cell lung cancer: a randomized, double-blind, placebo-controlled trial. Clin Cancer Res. 2018;24: 4089-97.

77. Schapochnik, Adriana, Marcia R. da Silva, Mayara P. Leal, Janete Esteves, Cristina B. Hebeda, Silvana Sandri, Daniela D. F. T. da Silva, Sandra H. P. Farsky, Rodrigo L. Marcos, and Adriana Lino-dos-Santos-Franco. 2018. Vitamin $D$ treatment abrogates the inflammatory response in paraquatinduced lung fibrosis. Toxicol Appl Pharmacol 355: 60-67.

78. Huang $Y$, Wang $L$, Jia X-X, Lin X-X, Zhang W-X. Vitamin $D$ alleviates airway remodeling in asthma by down-regulating the activity of Wnt/ $\beta$-catenin signaling pathway. Int Immunopharmacol. 2019;68:88-94.

79. Wang M, Liu M, Wang C, Xiao Y, An T, Zou M, Cheng G. Association between vitamin D status and asthma control: a meta-analysis of randomized trials. Respir Med. 2019;150:85-94.

80. Yu-Ying Q, Xiao-Yan Z, Xiu-Fen Q, Wu Y-X, Chu Q, Tao B. 1,25dihydroxyvitamin D3 reduces mouse airway inflammation of neutrophilic asthma by transcriptional modulation of interleukin-17A. Am J Transl Res. 2017;9:5411-21

81. Chambers ES, Nanzer AM, Pfeffer PE, Richards DF, Timms PM, Martineau AR, Griffiths CJ, Corrigan CJ, Hawrylowicz CM. Distinct endotypes of steroidresistant asthma characterized by IL-17Ahigh and IFN-phigh immunophenotypes: potential benefits of calcitriol. J Allergy Clin Immunol. 2015;136:628-37 e624.

82. Bakaletz LO, Novotny LA. Nontypeable Haemophilus influenzae (NTHi). Trends Microbiol. 2018;26:727-8.

83. Clarke C, Bakaletz LO, Ruiz-Guiñazú J, Borys D, Mrkvan T. Impact of protein D-containing pneumococcal conjugate vaccines on non-typeable Haemophilus influenzae acute otitis media and carriage. Expert Review Vaccines. 2017;16:751-64.

84. Zhang W, Zhang X, Sheng A, Weng C, Zhu T, Li C, Zhao W. Gammasecretase inhibitor alleviates acute airway inflammation of allergic asthma in mice by down-regulating Th17 cell differentiation. J Allergy Clin Immunol. 2015;135:AB225.

85. Kianmehr M, Haghmorad D, Nosratabadi R, Rezaei A, Alavinezhad A, Boskabady $\mathrm{MH}$. The effect of Zataria multiflora on Th1/Th2 and Th17/T regulatory in a mouse model of allergic asthma. Front Pharmacol. 2017;8: 458.

86. Morishima Y, Ano S, Ishii Y, Ohtsuka S, Matsuyama M, Kawaguchi M, Hizawa N. Th17-associated cytokines as a therapeutic target for steroid-insensitive asthma. Clin Dev Immunol. 2013;2013:1-9.

87. Busse WW, Holgate S, Kerwin E, Chon Y, Feng JY, Lin J, Lin S-L. Randomized, double-blind, placebo-controlled study of brodalumab, a human anti-IL-17 receptor monoclonal antibody, in moderate to severe asthma. Am J Respir Crit Care Med. 2013;188:1294-302.

88. Clancy R, Murree-Allen K, Cripps A, Yeung S, Engel M. Oral immunisation with killed Haemophilus influenzae for protection against acute bronchitis in chronic obstructive lung disease. Lancet. 1985;326:1395-7.

89. Clancy RL, Dunkley M. Acute exacerbations in COPD and their control with oral immunization with non-typeable Haemophilus influenzae. Front Immunol. 2011;2:7.

90. Mapp CE, Fryer AA, De Marzo N, Pozzato V, Padoan M, Boschetto P, Strange RC, Hemmingsen A, Spiteri MA. Glutathione S-transferase GSTP1 is a susceptibility gene for occupational asthma induced by isocyanates. J Allergy Clin Immunol. 2002;109:867-72.

91. Duan L, Li J, Ma P, Xu Y, Shunqing X. Vitamin E antagonizes ozone-induced asthma exacerbation in Balb/c mice through the Nrf2 pathway. Food Chem Toxicol. 2017:107:47-56

92. Emilie S, Anna C, Ignacio G-D, Giao D, Camille H, Canelle M, Catherine D, Carolyn H, Ingram John R, Luigi N, Olivier C, Laurence LC. Systemic pharmacological treatments for chronic plaque psoriasis: a network metaanalysis. Cochrane Database Syst Rev. 2017;12:CD011535.

93. Kenneth V, Frank P, Harald B, Juan L, Dries VR, Ann D, Isabel V, Kris P, Jan T, Lambrecht Bart N, Hamida H, Hans DW, Rudi B, Guy L, Savvides SN. Structure and antagonism of the receptor complex mediated by human TSLP in allergy and asthma. Nat Commun. 2017;8:14937.

94. Corren Jonathan, Parnes Jane R, Wang Liangwei, Mo May, Roseti Stephanie L, Griffiths Janet M, and van der Merwe René. Tezepelumab in adults with uncontrolled asthma. N Engl J Med. 2017;377:936-46.

95. He $Y$, Jingjing $S$, Tam NQ, Erli $Y$, Hongbo L, Xin R, Wu Z, Jianshuang $L$, Wenli Q, Kean KS, Yang T, Wei Y, Feng S, Zhijian X, Xiaozhu H, Karsten M, Booki M, Eric XH. Development of highly potent glucocorticoids for steroid-resistant severe asthma. Proc Natl Acad Sci U S A. 2019;116:6932-7.

96. O'Byrne Paul M, Hristo M, Margareta P, Kai R, Christina K, Mohib U, Bengt L, Marie C, Parameswaran N. Efficacy and safety of a CXCR2 antagonist, AZD5069, in patients with uncontrolled persistent asthma: a randomised, double-blind, placebo-controlled trial. Lancet Respir Med. 2016;4:797-806.

\section{Publisher's Note}

Springer Nature remains neutral with regard to jurisdictional claims in published maps and institutional affiliations.
Ready to submit your research? Choose BMC and benefit from:

- fast, convenient online submission

- thorough peer review by experienced researchers in your field

- rapid publication on acceptance

- support for research data, including large and complex data types

- gold Open Access which fosters wider collaboration and increased citations

- maximum visibility for your research: over $100 \mathrm{M}$ website views per year

At $\mathrm{BMC}$, research is always in progress.

Learn more biomedcentral.com/submissions 